\title{
INFORMATIONEN
}

Die Informationen in den folgenden Rubriken beruhen auf

Meldungen der mathematischen Institute/Fachbereiche.

\section{NEUE MITGLIEDER}

Petra Baumann, Zürich

Dr. Thomas Belzner, Poing

Julia Büsselberg, Berlin

Carerina Cozzi, Weißenhorn

Timo Dörzbach, Schriesheim

Prof. Dr. Gernot Fabeck, Schweinfurt

Daniela Fußeder, München

Leander Girrbach, Wilhelmsfeld

Christopher Herting, Markt Indersdorf

Collins Igbokwe, Weingarten

Dr. Janin Jäger, Fürth

Dr. Robert Lasarzik, Berlin

Dr. Judith Ludwig, Heidelberg

Georg Meinhardt, Bonn

Paul Mork, Aachen

Dr. Jens-Peter Müller, Differdange

Anna Maria Nölle, Konz

Jonas Peteranderl, München

Dr. Josef Rammensee, Regensburg

Dr. Aniko Ramshorn-Bircsak, Berlin

Maximilian Rettinger, Darmstadt

Tom-Christian Riemer, Chemnitz

Thore Johann Rümper, Loxstedt

Daniel Schiller, Oftersheim

Dr. Christian Schorr, Tholey

Max-Georg Schorr, Hannover

Prof. Dr. Stefan Schröer, Neuss

Lucas Schütt, Karlsruhe

Steve Schwarzkopf, Greiz

Dr. Jonathan Spreer, Sydney, Australien

Madeleine Todt-Kruse, Laage OT Klein Lantow

Patrick Uftring, Kahl am Main

Dr. Jonas Wahl, Bonn

Prof. Dr. Karl Worthmann, Ilmenau

Prof. Dr. Jane Zima, Runkel

\section{TODESFÄLLE}

Herr Prof. em. Dr. Heinz-Wilhelm Alten (Hildesheim) ist am 27. 1. 2019 verstorben.
Herr Prof. em. Dr. Klaus Heiner Kamps (Losheim am See) ist am 21. 2. 2019 verstorben.

Herr Heinz-Jürgen Hülsdunk (Schmitten/Ts.) ist am 23.12. 2019 verstorben.

Herr Dr. Eberhard H.-A. Gerbracht (Gifhorn) ist am 2. 2. 2020 verstorben.

Herr Dr. Jörg Brenner (Leonberg) ist am 16.4. 2020 verstorben.

Herr Prof. em. Dr. Dr. h.c. Heinrich Wefelscheid (Essen) ist am 18. 4. 2020 verstorben.

\section{HABILITATIONEN}

Tölle, Jonas M. (Universität Augsburg): Stochastic partial differential equations with singular drift. Beck, Blömker, Röckner, 15.01 .2020

\section{PROMOTIONEN}

Universität Bremen

Yang, Jichen: Diffusion, Advection and Pattern Formation. Rademacher, Kühn 23.03.20.

Etmann, Christian: Double Backpropagation with Applications to Robustness and Saliency Map Interpretability. Maaß, Möller 14.02.2020.

Westphal, Max: Model Selection and Evaluation in Supervised Machine Learning. Brannath, Zapf 16.12.2019.

Gerken, Thies: Dynamic Inverse Problems for Wave Phenomena. Rieder, Schmidt 07.10.2019.

\section{Technische Universität Dortmund}

Lohmann, Christoph: Physics-compatible finite element methods for scalar and tensorial advection problems. Kuzmin, Möller o8.05.2019.

Schulmann, Victor: Estimation of Stopping Times for Some Stopped Random Processes. Voit, Woerner 09.05.2019.

Pessik, Andre: Limit Theorems for Multipower Variations of Lévy Driven and Fractional-Lévy-Motion Driven Processes. Woerner, Dehling 28.05.2019.

Rosin, Korinna Kristina: Adaptive Finite Element Methods for Contact Problems embedded in a Fictitious Domain Simulation and Optimal Control. Rademacher, Wollner 24.06.2019.

Dröse, Jennifer: Textaufgaben lesen und verstehen lernen - Entwicklungsforschungsstudie zur mathematikspezifischen Leseverständnisförderung. Prediger, Schukajlow-Wasjutinski 24.06.2019.

Korten, Laura: Interaktiv-kooperative Lernsituationen im inklusiven Mathematikunterricht der Grundschule Entwicklungsforschungsstudie zum individuell-zieldifferenten Lernen am gemeinsamen Lerngegenstand des flexiblen Rechnens in interaktiv-kooperativen Lernsituationen. Selter, Wember, Höveler . 
DMV-Ansprechpartner/innen vor Ort

- Aachen: Gabriele Nebe - Augsburg: Bernhard Hanke - Bamberg: Anna Susanne Steinweg - Bayreuth: Thomas Peternell - FU Berlin: Ehrhard Behrends - FU Berlin: Günter M. Ziegler $-H U$ Berlin: Jürg Kramer - TU Berlin: Martin Skutella - WIAS Berlin: Wolfgang König - FH Bielefeld: Claudia Cottin $\square$ U Bielefeld: Michael Röckner $\square$ Bochum: Peter Eichelsbacher - Bonn: Daniel Huybrechts — Braunschweig: Volker Bach ॥ U Bremen: Anke Pohl ॥ Chemnitz: Christoph Helmberg — Cottbus: Friedrich Sauvigny - Darmstadt: Stefan Ulbrich — Dortmund: Ben Schweizer — Dresden: Andreas Thom - Duisburg: Rüdiger Schultz n Düsseldorf: Kai Köhler - Erlangen: Günter Leugering = Essen: Hans Niels Jahnke a Flensburg: Hinrich Lorenzen — Frankfurt am Main: Thorsten Theobald — Freiberg: Michael Eiermann — Freiburg: Sebastian Goette - Garching bei München: Peter Gritzmann — Gießen: Thomas Bartsch — Greifswald: Michael Schürmann — Göttingen: Thomas Schick $\square$ Hagen: Winfried Hochstättler $\square$ Halle: Rebecca Waldecker — TU Hamburg: Wolfgang Mackens - U Hamburg: Benedikt Löwe - Hannover: Christine Bes- senrodt - Heidelberg: Gebhard Böckle $\approx$ Hildesheim: Jürgen Sander n Hohenheim: Georg Zimmermann — Ilmenau: Carsten Trunk — Jena: Tobias Oertel-Jäger — Karlsruhe: Michael Plum - Kassel: Wolfram Koepf - Konstanz: Oliver Schnürer $\approx$ Köln: Peter Littelmann - Landshut: Konstantin Ziegler — Leipzig: Hans-Bert Rademacher — Lübeck: Jürgen Prestin — Lüneburg: Silke Ruwisch — Magdeburg: Volker Kaibel - Mainz: Martin Hanke-Bourgeois — Mannheim: Leif Döring = Marburg: Volkmar Welker — LMU München: Helmut Schwichtenberg — Münster: Michael Joachim n Neubiberg: Cornelius Greither - Neubrandenburg: Gerd Teschke = Oldenburg: Daniel Grieser — Osnabrück: Holger Brenner — Paderborn: Margit Rösler — Passau: Brigitte Forster-Heinlein $\square$ Potsdam: Christian Bär — Regensburg: Guido Kings — Rostock: Roger Labahn — Saarbrücken: Jörg Eschmeier — Siegen: Thorsten Raasch - HfT Stuttgart: Peter Hauber $-U$ Stuttgart: Timo Weidl - Trier: Jochen Wengenroth — Tübingen: Carla Cederbaum — Ulm: Günter Gramlich — Weimar: Klaus Gürlebeck - Wuppertal: Jens Hornbostel n Würzburg: Stefan Waldmann
Artykov, Merdan: Limit theorems for random walks on noncompact Grassmann manifolds with growing dimensions. Nührenbörger, Häsel-Weide 27.08.2019.

Rosnau, Timo: Asymptotic Class Numbers of Lattices. Scharlau, Zeiner 04.09.2019.

Mertens, Nick: Relaxation Refinement for Mixed-Integer Nonlinear Programs with Applications in Engineering. Buchheim, Liers 15.11.2019.

\section{Friedrich-Schiller-Universität Jena}

Wirth, Melchior: Entropic Gradient Flow Structure of Quantum Markov Semigroups. Lenz, Carlen, Maas 23.03.2020.

Kotrbat., Jan: Integral Geometry on the the Octonionic Plane. Wannerer, Bernig, Solanes 23.03.2020.

Sell, Daniel: Simple Toeplitz Subshifts: Combinatorial Properties and Uniformity of Cocycles. Lenz, Damanik, Baake 26.02.2020.

Lang, Julius: Three Projective Problems on Finsler Surfaces. Matveev, Muzsnay, Kruglikov 06.02.2020.

\section{Karlsruher Institut für Technologie}

Karrer, Annette: Contracting boundaries of amalgamated free products of CAT(o) groups with applications for right-angled Coxeter groups. Schwer, Hartnick 17.03.2020.

Möhler, Verena: SL(2,q)-Unitals. Herrlich, Stroppel 17.03.2020.

Schilling, Judith: Untersuchungen zur Asymptotik und zum Erwartungswert im verallgemeinerten Coupon-Collector-Problem. Henze, Hug 11.03.2020.

Karg, Christoph: On the Coarse Geometry of Regular Translation Surfaces. Weitze-Schmithüsen, Herrlich, Alessandrini 04.03.2020.

Otto, Moritz: Extremale Eigenschaften zufälliger Mosaike und Graphen. Last, Hug, Basrak 19.02.2020.

Leimcke, Gregor: Bayesian Optimal Investment and Reinsurance to Maximize Exponential Utility of Terminal Wealth. Bäuerle, Schmidt 22.01.2020.
Ziegler, Daniel: A Parallel and Adaptive Space-Time Discontinuous Galerkin Method for Visco-Elastic and Visco-Acoustic Waves. Wieners, Dörfler, Rieder 20.11.2019.

Garcia Perez, Ana Karla: Spaces and Moduli Spaces of Flat Riemannian Metrics on Closed Manifolds. Tuschmann, Sauer 13.11.2019.

Hagemann, Felix: Reconstructing the Shape and Measuring Chirality of Obstacles in Electromagnetic Scattering. Arens, Hettlich, Griesmaier 06.11.2019.

Grathwohl, Christine: Seismic Imaging with the Elliptic Radon Transform in 3 D: Analytical and Numerical Aspects. Rieder, Kunstmann, Schnaubelt 06.11.2019.

Flohr, Robin: Approximation of Traveling Waves by Splitting Methods. Jahnke, Plum 30.10.2019.

Schwarz, Sebastian: Fractional order splitting for semilinear evolution equations. Weis, Schnaubelt 17.10.2019.

Scheider, Dominic: On a Nonlinear Helmholtz System (Über ein nichtlineares Helmholtz-System). Mandel, Reichel, Weth 16.10.2019.

Cramer, Elena: Scattering of time-harmonic electromagnetic waves involving perfectly conducting and conductive boundary conditions. Kirsch, Hettlich 16.10.2019.

Geyer-Schulz, Andreas: Maxwell-Schrödinger System: Wellposedness and Standing Waves. Schnaubelt, Hundertmark 09.10.2019.

\section{Eberhard Karls Universität Tübingen}

Walach, Hanna Maria: Time integration for the dynamical low-rank approximation of matrices and tensors. Lubich 24.05.2019.

Manca, Benedetto: DPW potentials for compact symmetric CMC surfaces in the 3-sphere. Pedit 27.06.2019.

Jahns, Sophia: Trapping of light in stationary spacetimes. Cederbaum 27.09.2019.

Boßmann, Lea: Effective dynamics of interacting bosons: Quasilow-dimensional gases and higher order corrections to the mean-field description. Teufel 11.10.2019.

Johne, Florian: Surgery for extended Ricci flow systems. Huisken 08.11.2019. 
Braun, Lukas Maximilian: Quotient Presentations of Mori Dream Spaces. Hausen 08.11.2019.

de Franca Silva, Fernanda Clara: Some estimates for fractional and Carleson operators and sparse domination of uncentered variational truncations. Nagel 27.01.2020.

Kreidler, Henrik: Contributions to the Koopman Theory of Dynamical Systems. Nagel 11.09.2019.
Schmidt, Julian: Interior-Boundary Conditions as a Direct Description of QFT Hamiltonians. Teufel 01.04.2019.

Julius-Maximilians-Universität Würzburg

Promkam, Ratthaprom: Hybrid Dynamical Systems: Modeling, Stability and Interconnection. Dashkovskiy, Wirth o7.12.2019.

\section{DEUTSCHE MATHEMATIKER-VEREINIGUNG}

- VORSTAND UND PRÄSIDIUM Präsident Prof. Dr. Friedrich Götze, Fakultät für Mathematik, Universität Bielefeld, Universitätsstraße 25, 33615 Bielefeld, Tel. +49.521 106-4774 goetze@ math.uni-bielefeld.de Vizepräsident Prof. Dr. Klaus Hulek, Institut für Algebraische Geometrie, Gottfried Wilhelm Leibniz Universität Hannover, Welfengarten 1, 30167 Hannover, Tel.+49.511762-3205 hulek@math.uni-hannover.de Schatzmeister Prof. Dr. Etienne Emmrich, Institut für Mathematik, MA 5-3, Technische Universität Berlin, Straße des 17. Juni 136, 10623 Berlin, Tel.+49.3031425745, emmrich@math.tu-berlin.de Schriftführer Prof. Dr. Daniel Grieser, Universität Oldenburg, Institut für Mathematik, Carl-von-Ossietzky-Straße 9-11, 26129 Oldenburg, Tel.+49.441.7983230 daniel.grieser@uni-oldenburg.de Herausgeber der Mitteilungen Prof. Dr. Sebastian Stiller (verantwortlich), Technische Universität Carolo-Wilhelmina zu Braunschweig, Universitätsplatz 2,38106 Braunschweig, sebastian.stiller@tu-bs.de Weitere Präsidiumsmitglieder - Prof. Dr. Ilka Agricola, Marburg (Nachwuchsförderung) - Prof. Dr. Bernhard Hanke, Augsburg (Gauß-Vorlesung) - Prof. Dr. Moritz Kaßmann, Bielefeld (Industrieund Unternehmenskontakte) - Prof. Dr. Wolfram Koepf, Kassel (Vorsitzender der Kommission Übergang Schule-Hochschule) - Prof. Dr. Frank Loose, Tübingen (Kontakte in Schule und Hochschule) - Prof. Dr. Guido Schneider, Stuttgart (Herausgeber des Jahresberichtes der DMV) . Matthias Lippert, Remscheid (Fragen von Schule und Lehrkräftebildung) . Prof. Dr. Günter M. Ziegler, FU Berlin (Leiter Medien- und Netzwerkbüro der DMV) - Mitgliedsbeitrag 2020 (inkl. Bezug der Mitteilungen und einer gewählten Zeitschrift, Ausnahme: Studierende und Schüler beziehen nur die Mitteilungen) " regulär EUR 105,00 " bis zur Vollendung des 30. Lebensjahres EUR 50,00 . ermäßigt für

IMPRESSUM Verleger Walter de Gruyter GmbH, Berlin/Boston, www.degruyter.com - Herausgeber Prof. Dr. Sebastian Stiller (verantwortlich), Institut für Mathematische Optimierung, Technische Universität Carolo-Wilhelmina zu Braunschweig, Universitätsplatz 2, 38106 Braunschweig, sebastian. stiller@tu-bs.de — Prof. Dr. Michael Joswig, Fakultät II - Mathematik und Naturwissenschaften, Institut für Mathematik, MA 6-2, TU Berlin, Straße des 17. Juni 136, 10623 Berlin, joswig@math.tu-berlin.de • Prof. Dr. Brigitte Lutz-Westphal, Institut für Mathematik, Freie Universität Berlin, Arnimallee 3, 14195 Berlin, brigitte.lutz-westphal@math.fu-berlin.de - Prof. Günter M. Ziegler, Institut für Mathematik, FU Berlin, Arnimallee 2, 14195 Berlin, ziegler@math.fu-berlin.de — Redaktion Christoph Eyrich, Silke Thiel, Thomas Vogt, mdmv@
Ehepaare und eingetragene Lebenspartnerschaften EUR 150,00 - ermäßigt für Studierende (Bachelor/Master/Diplom) und Schülerinnen und Schüler EUR 20,00 . Sonderbeitrag auf Antrag (z. B. bei Arbeitslosigkeit) EUR 30,00 . ermäßigt für Mitglieder der DPG/GI/GOR/GDM/MNU oder MUED EUR 9o,oo EUR - ermäßigt für Reziprozitätsmitglieder (im Ausland wohnend und Vollmitglied einer Mathematischen Gesellschaft, mit der die DMV ein Reziprozitätsabkommen hat) EUR 70,0o - ermäßigt für Senioren EUR 70,00 — Zeitschriften (Jahresabo 2020 jeweils EUR 28,oo), eine der folgenden Zeitschriften ist im Mitgliedsbeitrag enthalten: - Jahresbericht der DMV (Springer Verlag Heidelberg, 4 Hefte jährlich) . Mathematische Semesterberichte (Springer Verlag Heidelberg, 2 Hefte jährlich) - Journal für MathematikDidaktik (Springer Verlag Heidelberg, 2 Hefte jährlich) — DMVServer www.mathematik.de - DOCUMENTA MATHEMATICA www.mathematik.uni-bielefeld.de/documenta/ @ Medienbüro der DMV Thomas Vogt, FU Berlin (mathematik.de) . Netzwerkbüro der DMV Thomas Vogt, FU Berlin (mathematik.de) - Geschäftsstelle der DMV Geschäftsführerin Andrea KirsteinGaekel (mathematik.de) - Bankverbindung Volksbank Freiburg 6955002 (BLZ 680 900 oo), IBAN: DE66 6809 oooo 00069550 o2, BIC: GENODE61FR1

Die Deutsche Mathematiker-Vereinigung e. V. ist durch den Körperschaftssteuerbescheid 2015 des Finanzamtes für Körperschaften Berlin I (Steuer-Nr. 27/640/51051) vom 16.5.2017, wegen „Förderung von Wissenschaft und Forschung" als wissenschaftlichen Zwecken dienend und zu den in $\S_{5}$ Absatz 1 Nr. 9 KStG bezeichneten Körperschaften gehörig anerkannt worden. Vereinseintrag: VR 380040 beim Amtsgericht Stuttgart. UmsatzsteuerIdentifikationsnummer: DE 165534138 .

math.tu-berlin.de a Adresse der Redaktion Mitteilungen der DMV, Institut für Mathematik, FU Berlin, Arnimallee 2, 14195 Berlin, Tel. +49.30.8387566o mdmv@math.tu-berlin.de - Grafische Gestaltung und Satz Christoph Eyrich, Berlin - Druck Grafisches Centrum Cuno, Calbe n Erscheinungsweise vierteljährlich. Der Bezugspreis ist im Mitgliedsbeitrag der DMV enthalten. Manuskripte senden Sie bitte an den Herausgeber.

Bitte senden Sie Adressenänderungen und alle die Mitgliedschaft betreffenden Zuschriften an die Geschäftsstelle der DMV, c/o WIAS, Mohrenstraße 39, 10117 Berlin, Tel. +49.30.20372-306, Fax +49.30.20372-307, dmv@wias-berlin.de I Namentlich gekennzeichnete Beiträge geben nicht unbedingt die Meinung der Redaktion wieder. 